\title{
High-Risk NAFLD among Patients with Irritable Bowel Syndrome: Frequency and Effect on Disease Severity
}

\section{Ahmed Samir Allam}

Faculty of Medicine, Ain Shams University, Cairo,Egypt https://orcid.org/0000-0002-0642-9911

Kadry Mohamed El Saeed

Faculty of Medicine, Ain Shams University, Cairo,Egypt

Hazem Mahmoud Abozeid

Faculty of Medicine, Ain Shams University, Cairo,Egypt

Khaled Mohamed Raafat ( $\square$ khaled.raafat@med.asu.edu.eg )

Faculty of Medicine, Ain Shams University, Cairo,Egypt

\section{Research Article}

Keywords: NAFLD, Irritable Bowel Syndrome, NAFLD fibrosis score, Lipid profile

Posted Date: January 12th, 2022

DOI: https://doi.org/10.21203/rs.3.rs-1252727/v1

License: (c) (i) This work is licensed under a Creative Commons Attribution 4.0 International License.

Read Full License 


\section{Abstract \\ Background}

Irritable bowel syndrome (IBS) is a functional gastrointestinal (GI) disease that can change a patient's quality of life and impair their daily activities. Non-alcoholic fatty liver disease (NAFLD), on the other hand, has become a widespread condition as the global obesity rates rises. The prevalence of NAFLD has reached up to $25 \%$ of the adolescent population. The etiology of both diseases is still not clearly understood. The mechanism linking the two seemingly similar diseases could be immune system activation and tissue inflammation; thus, the goal of our study was to see if there was a common link between them and to examine NAFLD prevalence and severity in IBS patients. Our study included 150 patients who have symptoms of IBS with different degrees of severity. IBS was diagnosed according to modified ROME IV criteria. Patients were examined to see if they had NAFLD based on abdominal ultrasonography and NAFLD fibrosis score calculation.

\section{Results}

Our current study showed that regarding evaluating the association of IBS with NAFLD, there was a highly statistically significant association between both diseases. Furthermore, there was a high statistical significant association between higher grades of NAFLD and lipid profile parameters.

\section{Conclusion}

Patients with IBS had a higher frequency of NAFLD. In addition, a significant association was noted between IBS severity and increased NAFLD grades.

\section{Introduction}

Irritable bowel syndrome (IBS) is a GI functional disorder that can affect people of any age, gender, race, or socioeconomic status. This condition has an important economic impact on patients because it usually lasts for life and impairs their quality of life. Furthermore, patients are more likely to seek medical attention and take time off from work. ${ }^{1}$ IBS molecular markers and drug targets are difficult to identify due to its variability and unidentified underlying causes. ${ }^{2}$

Irritable bowel syndrome (IBS) is defined by abdominal pain or discomfort, which is commonly linked with changes in bowel movements. IBS affects a large percentage of the general population (between $10 \%$ and $15 \%$ ). Women are more likely than men to suffer from IBS. ${ }^{3}$ Four IBS subtypes have been described based on stool features: diarrhoea (IBS-D), constipation (IBS-C), mixed (IBS-M), and undetermined (IBS-U) (IBS-U). Low-grade inflammation, altered motility, alterations in intestinal barriers, changes in gut-brain axis, and psychosocial variables appear to play a role in IBS pathophysiology. ${ }^{4}$ 
Nonalcoholic fatty liver disease (NAFLD) is a wide term that refers to a variety of disorders marked by fat deposition in hepatocytes in the presence or absence of liver inflammation. NAFLD refers to a group of fatty liver diseases that range from simple hepatosteatosis (HS), also known as nonalcoholic fatty liver (NAFL), to nonalcoholic steatohepatitis (SH), also known as NASH, and finally to liver cirrhosis. ${ }^{5}$

The main cause of NAFLD is obesity and increased body mass index (BMI), together with insulin resistance, in the absence of secondary causes of hepatic steatosis such as alcohol consumption, chronic use of medications that can cause hepatic steatosis, or hereditary disorders. ${ }^{6}$ NAFLD is diagnosed through imaging, blood tests including liver function test, different scoring systems; nonetheless, liver biopsy remains the most accurate test. ${ }^{7}$

In the diagnosis of NAFLD patients, a variety of scoring systems based on different clinical and/or laboratory characteristics have been developed. One of the commonly used is the NAFLD fibrosis score. This score depends on six different variables, which are age, hyperglycemia, body mass index (BMI), platelet count, albumin level, and AST/ALT ratio ${ }^{8}$

Obesity, gut microbiota dysfunction, a compromised intestinal barrier, and brain-gut axis dysfunction, all of which are crucial to their pathogenesis and are linked to immune activation and inflammation, may play a role in the development of IBS and NAFLD, but there aren't enough studies to back up this theory. ${ }^{9}$ As a result, the purpose of our research was to determine if there is a link between them and to investigate the prevalence and severity of NAFLD in IBS patients.

\section{Aim Of The Study}

The study's aim is to see if there is a link between IBS and NAFLD, as well as to determine the prevalence and severity of NAFLD in IBS patients.

\section{Subjects And Methods}

This study is a cross-sectional study conducted at the Gastroenterology and Hepatology Department of Ain Shams University Hospitals. It was conducted on 150 patients who had symptoms suggestive of IBS after excluding the following groups of patients: patients with Infectious Gastroenteritis or IBD, patients with cancer colon and patients with other viral (B and C) or alcoholic, genetic, or liver affections.

All cases underwent a full medical history with a focus on: abdominal pain frequency, change in bowel habits, and a family history of similar conditions. Patients were diagnosed with IBS by modified ROME IV criteria, which are: Recurrent abdominal pain on average at least 1 day/week in the last 3 months, associated with two or more of the following criteria: related to defecation, associated with a change in the frequency of stool, and associated with a change in the form (appearance) of stool. (These criteria must be met for the previous three months, with symptom onset at least six months preceding diagnosis.) ${ }^{10}$. 
Grading of IBS severity (according to the IBS severity grading score) was done and was classified into: Mild, could be ignored with effort but would have no effect on daily activities; moderate, could not be ignored, and occasionally interfered with daily activities; Severe, it could not be ignored and often limited concentration and daily activities. ${ }^{11}$

Additionally, a full clinical examination was done followed by laboratory investigations that included CBC, INR, AST, ALT, Na, K, Creatinine, and Urea.

The severity of NAFLD and the presence of liver fibrosis were also assessed using abdominal ultrasound and the NAFLD fibrosis score, with the diagnosis of NAFLD being confirmed by the presence of areas of high echogenicity on sonography. The severity of echogenicity was graded as follows: Grade 1: A mild, widespread increase in fine echoes in the liver parenchyma, with normal diaphragm and intrahepatic vascular boundaries; Grade 2: There is a modest, diffuse increase in fine echoes with slightly decreased visibility of intrahepatic veins and diaphragm; Grade 3: Increased fine echoes with poor or nonvisualization of intrahepatic vascular boundaries, diaphragm, and liver's posterior right lobe. ${ }^{12}$

NAFLD fibrosis score was also calculated for all patients. It was calculated as per the following formula: $-1.675+0.037 \mathrm{X}$ age (years) $+0.094 \mathrm{X}$ body mass index $(\mathrm{BMI}, \mathrm{kg} / \mathrm{m} 2)+1.13 \mathrm{X}$ impaired fasting glucose/diabetes (yes $=1$, no $=0)+0.99 X$ AST/ALT ratio $-0.013 X$ platelet $(X 109 / L)-0.66$ X Albumin $(\mathrm{g} / \mathrm{dL})$. The result was interpreted as low NFS $(<-1.445)$, indeterminate NFS $(-1.445$ to 0.676$)$ and high NFS $(>0.676)^{13}$

Statistical analysis data was collected and analyzed using SPSS software version 18 windows 7 . For quantitative variables, mean, standard deviation (SD), and range are used. Numbers and percentage are used for qualitative variables. Chi-square test was used to compare qualitative variables between groups. Power of significance was evaluated as follows: Probability level (P-value) $\geq 0.05$ : Insignificant, $P$-value < 0. 05: Significant, P-value < 0. 01: Highly significant.

\section{Results}

Table (1): Demographic data of included patients

\begin{tabular}{|lllll|}
\hline & Mean & SD & Median & Range \\
\hline Age (years) & 42.17 & 8.38 & 46 & $21-50$ \\
\hline Height (cm) & 173.62 & 9.07 & 175 & $155-189$ \\
\hline Weight (Kg) & 88.02 & 14.9 & 88 & $60-120$ \\
\hline BMI & 29.39 & 4.42 & 29.7 & $18.4-38.3$ \\
\hline Gender & Male & $74(49.3 \%)$ & \\
\cline { 2 - 5 } & Female & $76(50.7 \%)$ & \\
\hline
\end{tabular}


Table (1) showed that our current study included 150 patients diagnosed with IBS (74 males and 76 females). The mean age of included patients was $42.17 \pm 8.3$ years. Mean height was $173.6 \pm 9.07 \mathrm{~cm}$, mean weight was $88.02 \pm 14.9 \mathrm{Kg}$ while mean BMl was $29.39 \pm 4.4$.

Table 2: Diagnostic criteria of IBS and NAFLD in included patients

\begin{tabular}{|lll|}
\hline IBS grade & \multicolumn{1}{|c|}{$\mathbf{1}$} & \multicolumn{1}{c|}{ 96(64\%) } \\
\cline { 2 - 3 } & $\mathbf{2}$ & $44(29.3 \%)$ \\
\cline { 2 - 3 } U/S grade & $\mathbf{3}$ & $10(6.7 \%)$ \\
& $\mathbf{1}$ & $82(54.7 \%)$ \\
\cline { 2 - 3 } & $\mathbf{2}$ & $64(42.7 \%)$ \\
\hline Fibrosis score & $\mathbf{3}$ & $4(2.7 \%)$ \\
\cline { 2 - 3 } & Mean \pm SD & $-2.62 \pm 1.27$ \\
\cline { 2 - 3 } & Median & -2.6 \\
\cline { 2 - 3 } & Range & $-5.50-0.31$ \\
\hline
\end{tabular}

U/S: Ultrasound, SD: Standard Deviation

Table 2 showed that on evaluation of IBS and NAFLD in studied patients, 96 (64\%) patients had grade 1 IBS, 44 (29.3\%) patients had grade 2, and 10 (6.7\%) patients had grade 3 IBS. Regarding NAFLD grade, 82 $(54.7 \%)$ patients had grade $1,64(42.7 \%)$ patients had grade 2 , and only $4(2.7 \%)$ patients had grade 3. NAFLD fibrosis score had a mean of $-2.62 \pm 1.27$ in included patients

Figure (1) showed that on evaluation of IBS in studied patients, 96 patients had grade 1 IBS, 44 patients had grade 2, and 10 patients had grade 3 IBS.

Figure (2) showed that on evaluation of NAFLD in studied patients, 82 patients had grade 1, 64 patients had grade 2 and only 4 patients had grade 3 .

Table (3): Comparison between IBS grade and NAFLD grade

\begin{tabular}{|llllll|}
\hline & \multicolumn{4}{l}{ IBS grade No (\%) } & P value \\
\cline { 2 - 5 } & $\mathbf{1}$ & $\mathbf{2}$ & $\mathbf{3}$ & \\
\hline \multirow{2}{*}{ NAFLD grade } & $\mathbf{1}$ & $42(28 \%)$ & $50(33.3 \%)$ & $4(2.7 \%)$ & 0.006 \\
\cline { 2 - 5 } & $\mathbf{2}$ & $38(25.3 \%)$ & $6(4 \%)$ & 0 & \\
\cline { 1 - 5 } & $\mathbf{3}$ & $2(1.3 \%)$ & $8(5.3 \%)$ & 0 & \\
\hline
\end{tabular}


Table (3) showed that regarding evaluating the association of IBS with NAFLD, there was a highly statistically significant association between both diseases.

Table (4): Comparison of liver enzymes regarding IBS grade

\begin{tabular}{|lllll|}
\hline IBS grade & \multicolumn{2}{l}{ Serum ALT } & \multicolumn{2}{c|}{ Serum AST } \\
\cline { 2 - 5 } & Mean & SD & Mean & SD \\
\hline 1 & 22.7 & 12.9 & 23.2 & 11.2 \\
\hline 2 & 24.8 & 10.1 & 21.8 & 8.1 \\
\hline 3 & 26.6 & 4.6 & 24.6 & 6.9 \\
\hline P value & 0.525 & & 0.239 & \\
\hline
\end{tabular}

Using One-way ANOVA test

Table (4) showed that the study of IBS grade regarding liver enzymes revealed that there was no significant statistical difference between grades of IBS.

Table (5): Comparison of liver enzymes regarding U/S grade in NAFLD

\section{Using One-way ANOVA test}

\begin{tabular}{|lllll|}
\hline U/S grade & \multicolumn{2}{c}{ Serum ALT } & \multicolumn{2}{c|}{ Serum AST } \\
\cline { 2 - 5 } & Mean & SD & Mean & SD \\
\hline 1 & 28.2 & 11.8 & 26.2 & 10.2 \\
\hline 2 & 27.8 & 9.3 & 26.5 & 7.8 \\
\hline 3 & 31.6 & 5.2 & 29.5 & 7.3 \\
\hline P value & 0.342 & & 0.542 & \\
\hline
\end{tabular}

Table (5) showed that the study of NAFLD grade regarding liver enzymes revealed that there was no significant statistical difference between grades of NAFLD.

Table (6): Comparison of lipid profile parameters regarding IBS grade 


\begin{tabular}{|lllllll|}
\hline IBS grade & \multicolumn{2}{c}{ Serum cholesterol } & \multicolumn{2}{c|}{ Serum TG } & \multicolumn{2}{c|}{ Serum LDL } \\
\cline { 2 - 7 } & Mean & SD & Mean & SD & Mean & SD \\
\hline 1 & 181.8 & 55.5 & 141.8 & 65.9 & 95.3 & 47.4 \\
\hline 2 & 202.5 & 36.9 & 136.5 & 49.8 & 104.5 & 40.5 \\
\hline 3 & 169.4 & 40.3 & 149.8 & 53.7 & 97.8 & 16.6 \\
\hline P value & 0.346 & & 0.914 & & 0.821 & \\
\hline
\end{tabular}

Table (6) showed that the study of lipid profile parameters in relation to IBS grade revealed no-significant association.

Table (7): Comparison of lipid profile parameters regarding U/S grade

\begin{tabular}{|lllllll|}
\hline \multirow{2}{*}{ NAFLD grade } & \multicolumn{2}{c}{ Serum cholesterol } & \multicolumn{2}{c|}{ Serum TG } & \multicolumn{2}{c|}{ Serum LDL } \\
\cline { 2 - 7 } & Mean & SD & Mean & SD & Mean & SD \\
\hline 1 & 184.2 & 37.7 & 134.2 & 57.1 & 95.5 & 41.6 \\
\hline 2 & 182.1 & 57.6 & 134.6 & 42.1 & 96.5 & 39.2 \\
\hline 3 & 251.5 & 101.1 & 295.5 & 27.6 & 148 & 60.5 \\
\hline P value & 0.167 & & 0.001 & & 0.246 & \\
\hline
\end{tabular}

Using One-way ANOVA test

Table (7) showed that the study of lipid profile parameters (serum cholesterol, triglycerides, and LDL) in relation to $\mathrm{U} / \mathrm{S}$ grade revealed a highly significant association between increased serum TG and severe NAFLD.

\section{Discussion}

Irritable bowel syndrome (IBS) is a functional gastrointestinal (GI) disorder which can affect all members of a society, regardless of age, sex, race or socioeconomic status ${ }^{10}$.

Traditionally labelled as a functional GI disorder without evident structural or pathological changes, new insights suggest a disturbed GI physiology with impairment of GI motor function, visceral sensation and secretion, all of them potential therapeutic targets to improve symptoms and quality of life of these patients ${ }^{14}$.

IBS is characterized by abdominal pain or discomfort, classically linked to changes in bowel habits. A high percentage $(10-15 \%)$ of the general population suffer from IBS. IBS affects more females than males ${ }^{3}$. 
Nonalcoholic fatty liver disease (NAFLD) is a type of fatty liver disease that can be linked to overeating and its consequences, such as weight gain, central obesity, insulin resistance, glucose intolerance, atherogenic dyslipidemia, and arterial hypertension (metabolic syndrome), especially in people who are genetically predisposed. For a strict definition of NAFLD, Significant (or excessive) alcohol usage, as well as other diseases, such as hepatitis $\mathrm{C}$, hepatitis B, alcohol-related liver disease, and haemochromatosis must be eliminated ${ }^{15}$.

The aim of this study is to detect if there is a prevalent association between these two diseases and the assessment of NAFLD prevalence and severity in IBS patients. There are no efficient studies that highlight the link between NAFLD and IBS.

Our study has involved 150 patients with different degrees of IBS syndrome and variable severity patterns of NAFLD. Using inclusion and exclusion criteria for selecting the patients; IBS diagnosed according to modified ROME IV criteria regardless of the type either constipation predominance or diarrhea predominance. While NAFLD was mainly detected using abdominal ultrasonography, and NAFLD fibrosis score, both were differentiated into three grades determining the severity of each and their correlation.

Our study revealed that females and males almost equally suffered from IBS where 74 (49.3\%) males and 76 (50.7\%) females and this disagrees with the study of Milić and Stimac. $2012^{3}$ who found that IBS affects more females than males.

Overweight has been defined as a body mass index (BMI) or equal to 30. BMI has been the most useful population-level measure to define overweight and obesity, because the measurement applies to both sexes and adults of all ages ${ }^{15}$

Our study revealed that most of the randomly selected patients who have IBS were obese with mean BMI of $29.39 \pm 4.4$. This could explain the increased prevalence of NAFLD in IBS patients in our study. This would agree with study of Younossi et al., $2016^{15}$ and Fan et al., $2017^{16}$ who confirmed that obesity increases the risk of NAFLD.

Our study showed that on evaluation of IBS in studied patients, most of our IBS patients were stage 1 regarding severity as 96 patients had grade 1 IBS, 44 patients had grade 2, and 10 patients had grade 3 IBS. This disagree with the study of Ida et al., $2017{ }^{17}$ who showed that most patients enrolled had moderate to severe IBS symptoms in the baseline period.

Dyslipidemia is known as a risk factor for NAFLD. Our study showed that the study of lipid profile parameters (serum cholesterol, triglycerides, and LDL) in relation to U/S grade revealed a highly significant association between increased serum TG and severe NAFLD. These results were similar to the study of Mansour et al. $2019{ }^{18}$ who found that in the NAFLD group, a significant relationship was observed with TG, but no significant relationship was seen between LDL and NAFLD. In our study there was no significant association between TC, LDL or HDL and severity of NAFLD. These results disagree 
with Santhoshakumari et al. $2017^{19}$ who found that patients with NAFLD had higher TC, LDL, and TG, and lower $\mathrm{HDL}$ as compared to the control group.

Our study showed that regarding evaluating the association of IBS with NAFLD, there was a highly statistically significant association between both diseases. And these results were similar to Younossi et al., $2016^{15}$ who in their study revealed a direct proportional increase between the severity of irritable bowel syndrome and the severity of NAFLD where they concluded that It was clear here from our results that nearly all the study population (IBS) have a degree of (NAFLD) which come in concordance with increasing NAFLD cases. Also, similar to our results, Ahmed et al., (2018) ${ }^{20}$ reported in a cross sectional study that $74 \%$ of the study population showed moderate to severe NAFLD which is higher than the average reported prevalence rate among general population.

This relation could be due to a disruption of the intestinal microbiota in patients with NAFLD, which leads to increased fat storage and steatosis. This is mostly caused by decreased polysaccharide to monosaccharide fermentation and inadequate metabolism of short-chain fatty acids, both of which contribute to the development of IBS through modulating intestinal motility and sensitivity. The imbalance of intestinal microbiota caused by NAFLD can result in a breakdown of the intestinal barrier and reduced intestinal immunological tolerance ${ }^{15}$.

In our study, when comparing of different IBS grades and liver enzymes, there was no significant statistical difference between grades of IBS and liver enzymes. These results goes against Kang et al., $(2016)^{21}$ who reported- in a retrospective epidemiological study- significantly higher prevalence of elevated ALT level and metabolic syndrome (MS) in IBS patients compared to the controls. The relationship remained statistically significant after controlling for potential confounding factors.

Based on the current study, we found that patients with IBS had higher frequency of NAFLD. Also, there is significant association between IBS and NAFLD severity. Thus, patients with IBS should be frequently monitored to assess the presence of NAFLD: frequent clinical evaluation, abdominal ultrasonography in addition to Lipid profile and liver functions tests assessment if needed. Also, we could not exclude the psychological element as a confounder which is needed to be taken into consideration in following studies, the study patients were not subjected to psychological assessment.

\section{Conclusion}

Patients with IBS had a higher frequency of NAFLD. Furthermore, a significant association was noted between IBS severity and increased NAFLD grades. Nevertheless, our results need to be confirmed by further trials with a larger sample size, but also, there are few studies as far as we know, to explore the impact of NAFLD on the severity of IBS.

\section{Declarations}


Ethics approval and consent to participate

All procedures performed in this study were in accordance with the ethical standards of Ain Shams University Research Committee and with the 1964 Helsinki declaration and its later amendments.

Ethics committee's reference number : 000017585.

Address: Faculty of Medicine, Ain Shams University, Cairo 11211, Egypt.

Informed written consent was obtained from each participant before enrolment in the study

-Consent for publication: Informed written consent to publish patient's data was signed by all participants prior to beginning of the research.

-Availability of data and material: The authors confirm that the data supporting the findings of this study are available within the article.

-Competing interests: There is no conflict of interest.

-Funding: The funders had no role in study design, data collection and analysis, decision to publish, or preparation of the manuscript.

-Authors' contributions: A.A.S., E.K.M., A.H.M. and R.K.M. conceived and planned the experiments.

A.H.M. contributed to sample preparation. All authors provided critical feedback and helped shape the research, analysis and manuscript. All authors have read and approved the manuscript.

-Acknowledgements: Not applicable

\section{Abbreviations}

IBS Irritable bowel syndrome

NAFLD Non-alcoholic fatty liver disease

HS. simple hepatosteatosis

$\mathrm{NASH}$ nonalcoholic steatohepatitis

$\mathrm{BMI}$ Body mass index

TC. serum Total cholesterol

TG. serum triglycerides

LDL serum low density lipoprotein 
HDL serum high density lipoprotein

NFS NAFLD fibrosis score

MS Metabolic syndrome.

U/S Ultrasound

GIT ..Gastrointestinal Tract

AST . . Aspartate Aminotransferase

ALT... Alanine Aminotransferase

INR International Normalized Ratio

$\mathrm{Na}$ Sodium

$\mathrm{K}$ Potassium

CBC Complete Blood Count

ELISA. ..Enzyme Linked Immunosorbent assay

ANOVA Analysis of variance

ROC-curve. Receiver Operating Characteristic curve

PPV Positive Predictive value

NPV. Negative Predictive value

AUC Area under curve

\section{References}

1. Shah SL, Janisch NH, Crowell M, et al. Patients with irritable bowel syndrome are willing to take substantial medication risks for symptom relief. Clin Gastroenterol Hepatol. 2021; 19: 80-86.

2. Gu Y, Zhou G, Qin X, Huang S, Wang B, Cao H. The potential role of gut mycobiome in irritable bowel syndrome. Frontiers in microbiology. 2019; 10:1894.

3. Milić S, Stimac D. Nonalcoholic fatty liver disease/steatohepatitis: epidemiology, pathogenesis, clinical presentation and treatment. Dig Dis. 2012;30(2):158-62.

4. CheyW, Kurlander J, Eswaran S. Irritable bowel syndrome: A clinical review. JAMA 2015, 313: 949958. 
5. Drescher HK, Weiskirchen S, Weiskirchen R. Current status in testing for nonalcoholic fatty liver disease (NAFLD) and nonalcoholic steatohepatitis (NASH). Cells. 2019;8:E845.

6. Bai T, Xia J, Jiang Y, Cao H, Zhao Y, Zhang L, Wang H, Song J, Hou X. Comparison of the Rome IV and Rome III criteria for IBS diagnosis: A cross-sectional survey. J. Gastroenterol. Hepatol. 2017; 32: 1018-1025.

7. Sadik R, Björnsson E, Simrén M. The relationship between symptoms, body mass index, gastrointestinal transit and stool frequency in patients with irritable bowel syndrome. Eur $\mathrm{J}$ Gastroenterol Hepatol 2010; 22: 102-108.

8. Leoni S, Tovoli F, Napoli L, Serio I, Ferri S, Bolondi L. Current guidelines for the management of nonalcoholic fatty liver disease: a systematic review with comparative analysis. World J Gastroenterol. 2018; 24:3361-3373.

9. Scalera A, Matteo NDM, and Giovanni T. "What does irritable bowel syndrome share with nonalcoholic fatty liver disease?." World Journal of Gastroenterology: WJG 2013; 19(33): 5402.

10. Mearin F, Lacy BE, Chang L, Chey WD, Lembo AJ, Simren M, Spiller R. Bowel disorders. Gastroenterology. 2016; 150(6):1393-407.

11. Hahn BA, Kirchdoerfer LJ, Fullerton S, Mayer E. Patient-perceived severity of irritable bowel syndrome in relation to symptoms, health resource utilization and quality of life. Aliment Pharmacol Ther 1997; 11: 553-559.

12. Pardhe, Shakya, Bhetwal, Mathias J, Khanal, Pandit, et al. Metabolic syndrome and biochemical changes among nonalcoholic fatty liver disease patients attending a tertiary care hospital of Nepal. BMC Gastroenterol 2018;18:109

13. Rinella $M<$ bi $>$.</bi> Non-alcoholic fatty liver disease: a systematic review313:2263-73. doi: 10.1001/jama.2015.5370.

14. Canavan, Caroline, Joe West, and Timothy Card. "The epidemiology of irritable bowel syndrome." Clinical epidemiology 6 (2014): 71.

15. Younossi ZM, Koenig AB, Abdelatif D, Fazel Y, Henry L, Wymer M. Global epidemiology of nonalcoholic fatty liver disease-meta-analytic assessment of prevalence, incidence, and outcomes. Hepatology 2016; 64: 73-84.

16. Fan JG, Kim SU, Wong VW. New trends on obesity and NAFLD in Asia. J Hepatol 2017;67:862-873.

17. Ida M., Akito Nishida, Hiraku Akiho, Yoshihiro Nakashima, Kei Matsueda and Shin Fukudo. Evaluation of the irritable bowel syndrome severity index in Japanese male patients with irritable bowel syndrome with diarrhea. BioPsychoSocial Medicine (2017) 11:7

18. Roya MansourGhanaei, Fariborz MansourGhanaei, Mohammadreza Naghipour, Farahnaz Joukar. Biochemical markers and lipid profile in nonalcoholic fatty liver disease patients in the PERSIAN Guilan cohort study (PGCS), Iran. 2019 Journal of Family Medicine and Primary Care. Volume 8: Issue 3 : March 2019-923-928.

19. Santhoshakumari TMJ, Radhika G, Kanagavalli P. A study of anthropometric and lipid profile parameters in non-alcoholic fatty liver disease patients attending a tertiary care hospital at 
puducherry. IOSR J Dent Med Sci (IOSR-JDMS) 2017;16:33-7.

20. Ahmed A, Perumpail BJ, Li AA, John N, Sallam S, Shah ND, Kwong W, Cholankeril G, Kim D. The Role of Vitamin $E$ in the Treatment of NAFLD. Diseases. 2018; 6(4):86.

21. Kang MK, Kang BH, Kim JH. Nonalcoholic fatty liver disease is associated with the presence and morphology of subclinical coronary atherosclerosis. Yonsei Med. J. 2016; 56: 1288-1295.

\section{Figures}

\section{IBS Grade}

120

100

80

60

40

20

0

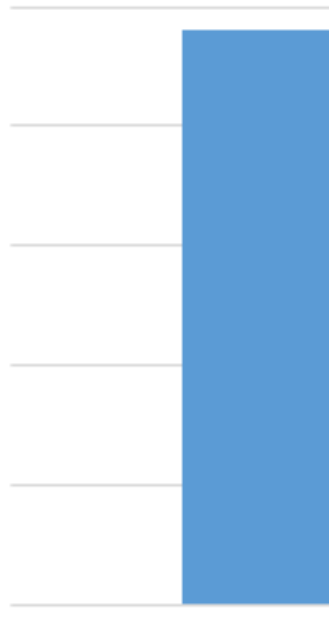

Grade 1

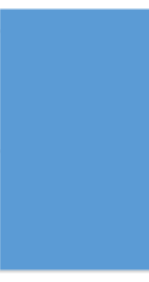

Grade 2

Grade 3

IBS Grade

Figure 1

IBS grade of included patient 


\section{U/S Grade}

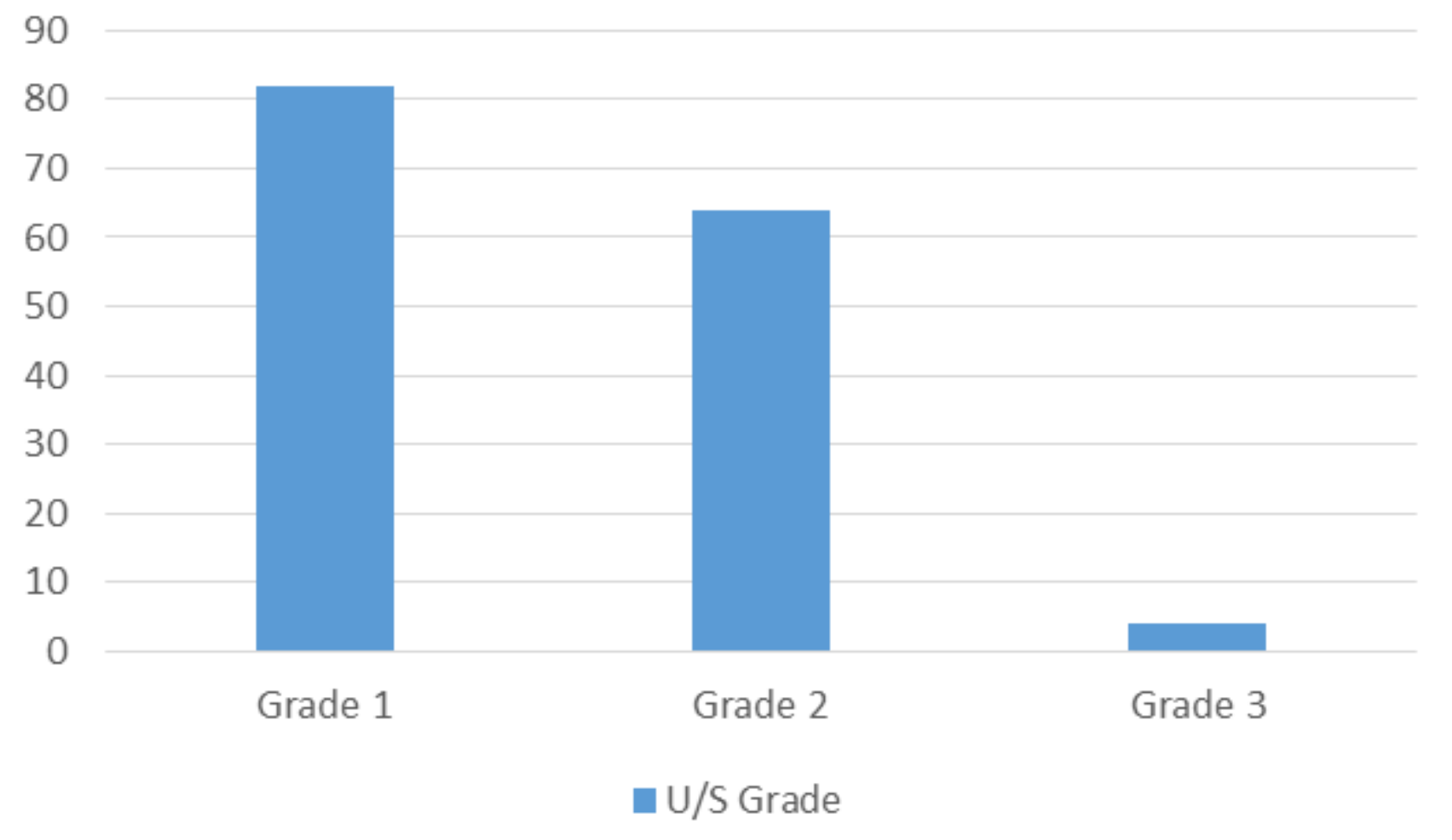

Figure 2

$\mathrm{U} / \mathrm{S}$ grade of included patient 